\title{
Multiobjective Neuroevolutionary Control for a Fuel Cell Turbine Hybrid Energy System
}

\author{
Mitchell Colby \\ Oregon State University \\ Corvallis, OR \\ colbym@engr.orst.edu \\ David Tucker \\ National Energy Technology \\ Laboratory Morgantown, WV \\ David.Tucker@netl.doe.gov
}

\author{
Logan Yliniemi \\ University of Nevada, Reno \\ Reno, NV \\ logan@unr.edu \\ Kenneth "Mark" Bryden \\ Ames Laboratory Ames, IA \\ kmbryden@iastate.edu
}

\author{
Paolo Pezzini \\ Ames Laboratory Ames, IA \\ ppezzini@ameslab.gov \\ Kagan Tumer \\ Oregon State University \\ Corvallis, OR \\ kagan.tumer@oregonstate.edu
}

\begin{abstract}
Increased energy demands are driving the development of new power generation technologies with high efficient. Direct fired fuel cell turbine hybrid systems are one such development, which have the potential to dramatically increase power generation efficiency, quickly respond to transient loads (and are generally flexible), and offer fast start up times. However, traditional control techniques are often inadequate in these systems because of extremely high nonlinearities and coupling between system parameters. In this work, we develop multi-objective neural network controller via neuroevolution and the Pareto Concavity Elimination Transformation (PaCcET). In order for the training process to be computationally tractable, we develop a computationally efficient plant simulator based on physical plant data, allowing for rapid fitness assignment. Results demonstrate that the multi-objective algorithm is able to develop a Pareto front of control policies which represent tradeoffs between tracking desired turbine speed profiles and minimizing transient operation of the fuel cell.
\end{abstract}

\section{CCS Concepts}

$\bullet$ Hardware $\rightarrow$ Fuel-based energy;

\section{Keywords}

Multi-objective optimization; PaCcET

\section{INTRODUCTION}

Increases in population as well as per capita energy consumption have driven the demand for energy sources which are thermally efficient, economically viable, and environmentally friendly. One potential class of solutions involves hybrid power generation systems, which combine existing

Permission to make digital or hard copies of all or part of this work for personal or classroom use is granted without fee provided that copies are not made or distributed for profit or commercial advantage and that copies bear this notice and the full citation on the first page. Copyrights for components of this work owned by others than ACM must be honored. Abstracting with credit is permitted. To copy otherwise, or republish, to post on servers or to redistribute to lists, requires prior specific permission and/or a fee. Request permissions from permissions@acm.org.

GECCO '16, July 20-24, 2016, Denver, CO, USA

(C) 2016 ACM. ISBN 978-1-4503-4206-3/16/07 . \$ $\$ 15.00$

DOI: http://dx.doi.org/10.1145/2908812.2908924 technologies resulting in synergistic relationships between components. In particular, research is currently being conducted on fuel cell turbine hybrid energy systems.

A key difficulty in fuel cell turbine hybrid systems is that the combination of recuperative turbine cycles and solide oxide fuel cells result in high nonlinearities as well as extreme coupling between state variables, making high fidelity models difficult to develop. Without accurate models, traditional control techniques such as optimal control or model predictive control become difficult to implement.

One potential solution is the use of model-free control techniques such as evolving controllers. While this does eliminate the need for a model, it introduces the problem of fitness assignment. Often, the performance of a controller needs to be determined in computationally expensive numerical simulations, or tested in hardware. In either case, the cost of fitness assignment results in evolutionary algorithms becoming computationally intractable.

In this work, we create an efficient statistical model of the Hybrid Performance Project (Hyper), a fuel cell turbine hybrid power plant located at the National Energy Technologies Laboratory in Morgantown, West Virginia. Using this model as the basis for fitness assignment, we evolve multi-objective neural network controllers for the facility.

The specific contributions of this paper are to:

- Model the behavior of an advanced fuel cell turbine hybrid energy system.

- Use multi-objective neuroevolutionary control algorithms to develop controllers for the Hyper facility.

Figure 1 shows an outline of the process used to develop a multiobjective neural network controller for Hyper, and is referred to in later sections of the paper as each component of the process is discussed.

The rest of this paper is organized as follows. Section 2 introduces Hyper, Section 3 details background and related work, Section 4 details the development of the simulator for Hyper, Section 5 discusses the multi-objective neuroevolutionary algorithm, Section 6 presents experimental results, and Section 7 concludes the paper.

\section{HYBRID PERFORMANCE PROJECT}

The following sections provide an overview of the Hybrid Performance Project and its current control techniques. 


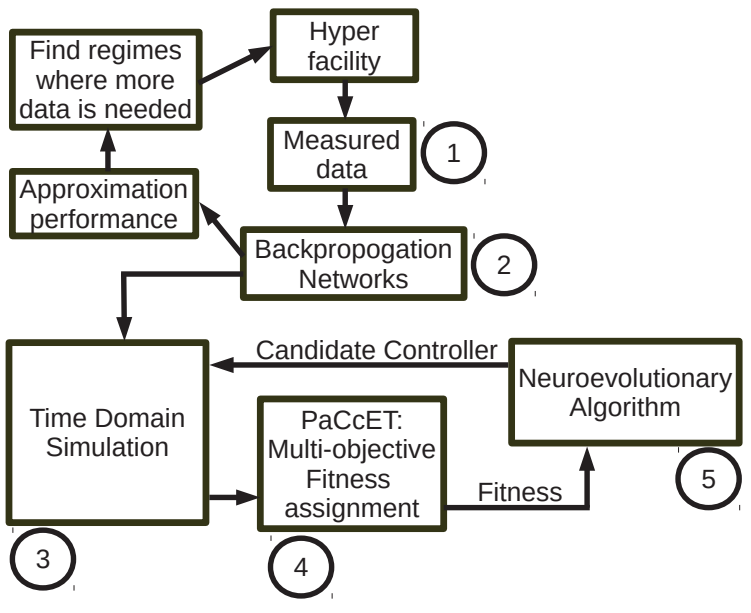

Figure 1: Flow chart of the approach for the multiobjective neuroevolutionary control of the Hyper facility.

\subsection{Hyper Overview}

The Hybrid Performance Project facility (Figure 2), Hyper, is located at the Department of Energy's National Energy Technology Laboratory (NETL) campus in Morgantown, West Virginia. The purpose of this experimental plant is to study the complex interactions of the direct fired Solid Oxide Fuel Cell (SOFC) gas turbine hybrid configuration, as well as to develop control strategies for such a system.

Hyper is a small scale SOFC gas turbine hyrbid hardware based simulation, capable of emulating 320 to $820 \mathrm{~kW}$ hybrid plants [19]. Hyper contains a hardware simulation of a $200 \mathrm{~kW}$ to $700 \mathrm{~kW}$ solid oxide fuel cell (SOFC) system coupled with a $120 \mathrm{~kW}$ turbine (Figure 2) [17].

The hardware based fuel cell simulation makes Hyper unique in that it allows for a wide range of fuel cells to be simulated without additional cost, and allows for testing of control strategies without risk of damaging a costly fuel cell. The fuel cell can be reset in software rather than being rebuilt in hardware, allowing for significant progress in the understanding of such hybrid configurations $[8,12,14]$.

The standard recuperative cycle is a fundamental building block of Hper. The recuperative cycle is a gas turbine cycle

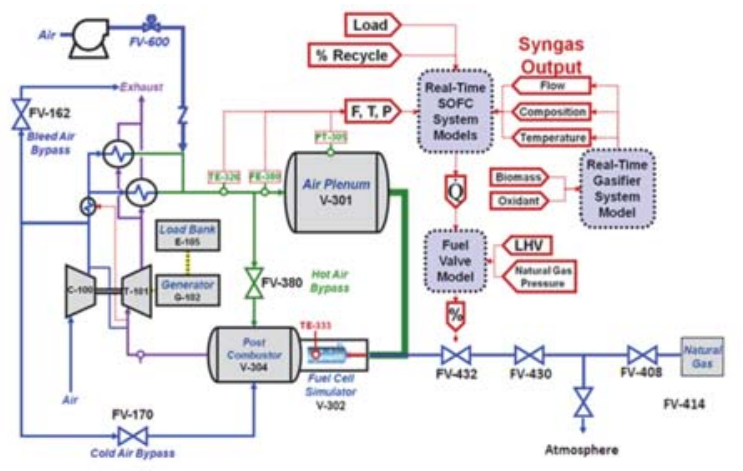

Figure 2: Diagram for Hybrid Performance Project facility at NETL. model used for power generation. Gas cycle turbines are effective in power generation because they have fast start up times, can be built for a wide range of power outputs, and can use readily available fuels such as natural gas [19].

Typically atmospheric air is drawn into the system through a compressor. The pressurized air is then mixed with fuel in a combustion chamber and the fuel is ignited by adding thermal energy to the air mixture. The exhaust gases from the combustion chamber are then expanded through a gas turbine, generating mechanical work in a rotating shaft used to drive the compressor and electric generator. A standard gas turbine then vents the exhaust gases from the turbine out into the atmosphere. In a turbine with regeneration, the hot exhaust from the turbine is used to preheat the air entering the combustion chamber with a heat exchanger, reducing the amount of fuel required to heat the air.

Solid oxide fuel cells utilize a ceramic electrolyte to channel oxygen ions to react with hydrogen, producing an electric current. Fuel cell turbine hybrids operate at very high efficiency, typically up to $60-75 \%$ and with low carbon emissions [21]. These fuel cells operate at a high temperature, reforming natural gas or other hydrocarbons to produce the hydrogen needed for the reaction, and ionizing the hydrogen and oxygen to be transported across the electrolyte. Temperatures can reach up to $1000^{\circ} \mathrm{C}$, much of which is wasted in the exhaust. Fuel cells are typically slow to heat and start up, limiting their use in applications requiring fast startup [19]. Pressurized air enters the cathode of the fuel cell, and fuel enters the anode. Hot exhaust leaves the fuel cell at a high temperature, along with any unconverted fuel.

The Hyper project places a hardware simulation of a fuel cell between the regeneration heat exchangers and combustion chamber of a typical regeneration cycle. Primary heat generation to run the turbine comes from the fuel cell exhaust. The combustion chamber burns any unspent fuel, assists in start up, and regulates turbine inlet temperature. Exhaust from the turbine runs through a set of parallel heat exchangers to preheat air into the fuel cell. Nearly 200 sensors are located across the plant designed to provide realtime information to the controller and log the system state during experiments.

In this work, we focus on the control of three bypass air valves, which can adjust the state of the Hyper system. These bypass valves (seen in Figure 2) are:

- Cold air bypass (FV-170): regulates cold air flow to the cathode of the fuel cell.

- Hot air bypass (FV-380): regulates flow of high pressure hot air from the heat exchangers to the combustion chamber.

- Bleed air valve (FV-162): regulates flow of high pressure air from the compressor to the atmosphere.

System wide analytic models and software simulations have yet to be perfected for the Hyper facility, limiting the study of different control studies for the plant. The following section discusses the current methods for controlling Hyper.

\subsection{Hyper Control Strategies}

Modern control theory was designed to deal with large, multi-input, multi-output systems, and is the basis for modern power plant control strategies. Historically, developing highly accurate models for traditional coal-fired power 
plants has been completed using thermodynamics, heat transfer, and fluid dynamics laws. Further, many model-based control strategies for power systems, including model predictive control, allow for some error in the system model without significantly affecting the performance of the control strategy [9]. As a result of the availability of relatively accurate plant models, model based control methods are prevalent in most power plants. These control strategies rely on two major assumptions:

1. (Reasonably) accurate models exist for the system.

2. Models can be linearized about a feasible operation point.

Several methods of modeling and control of Hyper have been used to provide a demonstration of the potential of hybrid fuel cell technology $[18,15]$. Most notably, an adaptive control scheme offers some solutions to the coupled, nonlinear control system in the Hyper facility [16]. Using a model reference adaptive control, the plant was shown to be controllable in simulation. This control strategy utilizes empirical transfer functions built from plant characterization tests as a non-linear model of the plant. A control loop uses these plant models to determine the optimal control input.

While empirical transfer functions of the plant contain information that captures coupling and nonlinearities in the system, these functions are extremely costly to acquire; developing these transfer functions require running multiple plant characterization experiments, where the frequency of actuation is varied and the plant state is captured and recorded. Further, if hardware changes are incorporated into the plant, the transfer functions are invalidated, requiring the plant characterization experiments to be rerun. The model reference adaptive control can also result in unwanted oscillations due to the nature of the empirical transfer functions. In this paper, we present a model-free control strategy to relax the requirement for a high-fidelity system model.

\section{BACKGROUND}

The following sections describe related work on multiobjective optimization, neuroevolutionary control, and fitness modeling.

\subsection{Multi-Objective Optimization}

With multiple simultaneous objectives to optimize, our definition of optimality must change to include not only the single-objective optima, but also the optimal tradeoffs between these extremes. This comes from the concept of Pareto optimality, which is a central solution concept in multi-objective optimization. A point is said to be Pareto optimal if it is not dominated by another solution, where domination is defined as follows. A solution $u$ dominates another solution $v(u \prec v)$ if it scores lower on all criteria (objectives $c$ ): $\forall c\left[f_{c}(u)<f_{c}(v)\right]$. A solution $u$ weakly dominates another solution $v(u \preceq v)$ if it scores equal on some objectives, but less on others: $\forall c\left[f_{c}(u) \leq f_{c}(v)\right] \wedge$ $\exists j \mid\left[f_{j}(u)<f_{j}(v)\right][20]$. The set of Pareto optimal solutions is termed the Pareto front.

Multi-objective optimization has the convention of minimization, which we use here but in this work we are maximizing the accuracy; to resolve this, one can consider the maximization problem as one of minimizing the negative accuracy without a loss of generality.

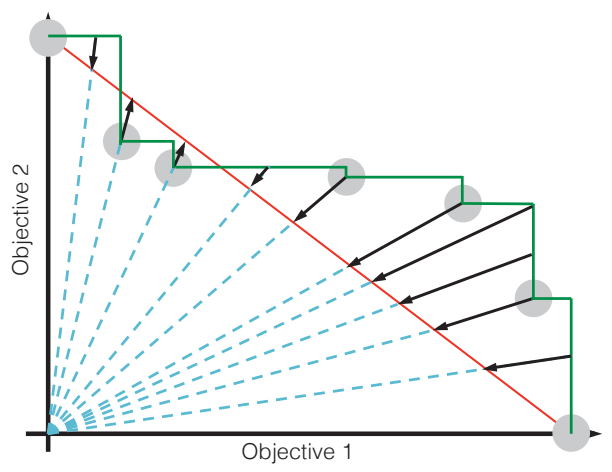

Figure 3: PaCcET maps the current-best-estimate of the Pareto front ( $P_{I}^{*}$, grey points) onto an equally valuable line to a linear combination (red line) by moving points radially away or toward the utopia point (origin in this figure). Non-dominated points are more valuable to a linear combination (than dominated or points in $P_{I}^{*}$ ) after the transform.

There are a host of multi-objective optimization algorithms, but in this work we limit our consideration to the Pareto Concavity Elimination Transformation (PaCcET, [22]) because of the following reasons: 1) It executes over an order of magnitude faster than some of the other state-of-the-art algorithms, 2) it has theoretical guarantees stating that the generated solutions will be Pareto optimal 3) It has been shown to function well in domains with arbitrary Pareto fronts and 4) it is agnostic to the optimizer used, and is typically not sensitive to optimizer parameters [22].

The primary function of PaCcET (Figure 3 ) is to constantly use the current best-estimate of the Pareto front $\left(P_{I}^{*}\right)$ as an approximation of the nonlinear preference function that would make every Pareto optimal solution equally valuable. PaCcET then projects these solutions onto a line that is equally valuable to a linear combination of (transformed) objectives, which moves all unattained areas of the objective space into the more valuable portion of the space. These solutions will then be preferred, and once attained, they will update the approximation of the Pareto front to be a better approximation of the true Pareto front. PaCcET, in this way, allows any single-objective optimizer to perform a multi-objective optimization, with these guarantees.

\subsection{Neuroevolutionary Control}

Neural networks as controllers have the capability to reject sensor and actuator noise, generalize to previously unseen system states, and can outperform traditional linear control methods for many difficult control problems $[5,10]$. Nonlinearities and coupling between state variables in the system make many problems difficult for traditional control techniques, as linearizations about operation points too coarsely approximate the system dynamics. Training these networks is possible when no explicit system model exists through neuroevolution [13].

Neuroevolutionary control has been shown to be successful in many difficult and highly nonlinear domains, including micro-air vehicle control in air currents [10], dynamic quadrotor flight [11], wave energy converter ballast design [4], and finless rocket altitude and attitude control [5].

In the context of power plant control, fitness assignment 
is difficult in neuroevolutionary algorithms; fitness of a controller cannot be evaluated on the physical plant due to safety considerations, and high fidelity numerical simulations are too slow for a computationally tractable search through the solution space. Thus, in order to develop controllers for power plants via neuroevolution, fitness assignment must be made to be computationally cheaper.

\subsection{Fitness Modeling}

In cases where the system performance cannot be directly modeled (or such a model is computationally expensive), the system performance can be approximated in order to provide feedback for an agent learning a control strategy for the system $[1,2]$. These techniques to approximate fitness functions are known as fitness modeling.

Fitness modeling has been shown to successfully allow for evolutionary algorithms to be computationally tractable in complex domains [3, 7]. Typically, the fitness evaluation dominates the computational cost of an evolutionary algorithm [6]. In the Hyper facility, hardware simulations run in real-time, and high fidelity simulations can run in near real-time. Thus, in order to develop control strategies for Hyper using neuroevolution, the time needed to evaluate a particular control policy must be dramatically reduced.

\section{HYPER SIMULATOR}

In order to evolve a controller for Hyper, the performance of a controller in Hyper must be evaluated with minimal computational cost. Thus, we develop a neural network based simulator for Hyper. There are two key difficulties in modeling Hyper. First, sensor data from experimental Hyper runs is noisy, so care must be taken to ensure the simulator does not overfit the data. Second, there exists extremely high coupling between state variables in Hyper, so the neural network based simulator must be designed to account for the coupling between state variables, while minimizing the effects of crosstalk within the networks.

By using real plant data from physical Hyper runs, we can ensure the simulation of the plant learns the underlying dynamics of the system. Once the neural network based simulator is developed, we can quickly evaluate different control policies, allowing for a neuroevolutionary algorithm to become computationally tractable. The following sections detail the process of developing the neural network based simulator for Hyper.

\section{Data Selection.}

To train a network that can simulate the plant, a suitable data set was found, corresponding to Box 1 in Figure 1. Many distinct experiments run at the Hyper facility, and data was collected and recorded to be used for a training set for the Hyper simulator. The chosen data set was a recent experiment involving a characterization of the cold air valve. In this experiment, the cold air valve was opened to a position and was systematically changed once the plant had reached steady state. The valve was changed between $10 \%$ and $80 \%$ open. The resultant data set contains 19 plant state variables, and 2 control variables.

The data set contained 30,626 data points sampled at $12.5 \mathrm{~Hz}$, about 10 minutes of run data. To mitigate potential problems with sensor noise affecting simulator training, data was down-sampled by averaging datapoints every 25 timesteps. Thus, 1225 points (at a corresponding frequency of $0.5 \mathrm{~Hz}$ ) are presented to the simulator to learn. All data points are then scaled between 0.1 and 0.9 , corresponding to the observed variable minimum value and maximum value respectively such that the input to the simulator is scaled consistently.

\section{Backpropagation.}

Once the data was collected, a neural network based simulator was trained to learn the state transition mapping $T\left(s_{t}, a_{t}\right) \rightarrow s_{t+1}$; in other words, the simulator maps a state $s_{t}$ and action $a_{t}$ at time $t$ to the resultant state $s_{t+1}$ at time $t+1$. It was determined that a single two-layer feedforward neural network was insufficient to accurately model the dataset, due to crosstalk in the first weight layer. To eliminate crosstalk, we developed an ensemble based simulator (Box 2 in Figure 1).

The Hyper training data contains $n=19$ state variables and $m=2$ control variables. Our simulator contains 19 neural networks, each of which maps the system state $s_{t} \in \mathbb{R}^{n}$ and action $a_{t} \in \mathbb{R}^{m}$ to a single state variable $s_{t+1}^{i} \in \mathbb{R}$. The ensemble is presented with a state $s_{t}$ and action $a_{t}$, and each neural network $N N_{i}$ finds a single value in the resultant state vector $s_{t+1}$. Each network was trained with backpropagation, with a learning rate of 0.2 and a momentum term of 0.5 . These parameters were chosen using a parameter sweep, but results are similar as long as the learning rate is below 0.4 (higher learning rates caused poor results). A single state transition from the neural network ensemble is shown in Algorithm 1.

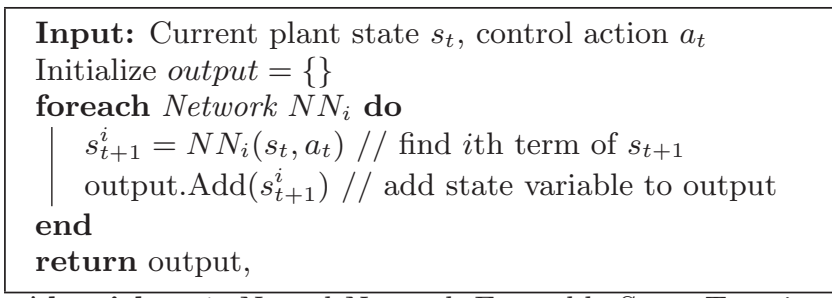

Algorithm 1: Neural Network Ensemble State Transition Mapping

After training the neural network ensemble, its approximation performance was evaluated to determine its effectiveness. We found areas of the state space where approximation error was high, and collected more data from physical Hyper runs to improve the resolution of training data in those operational regimes; data from a plant startup experiment was added to the training data set. This active data collection process is shown in the data collection loop in Figure 1.

Once the training data set was finalized, the simulator training process was repeated for 25 statistical trials to demonstrate the statistical significance of the training algorithm. For each statistical run, 100 data points were randomly removed from the training set to create a validation set. The resulting mean squared error on the validation set had an average of $0.12 \%$ with a variance of $0.02 \%$. The maximum error for each state variable had an average of $1.71 \%$ with a variance of $0.11 \%$.

\subsection{Time Domain Simulation}

The best network ensemble found during the training process described above is used to develop a time domain simulation of Hyper, corresponding to Box 3 in Figure 1. The 


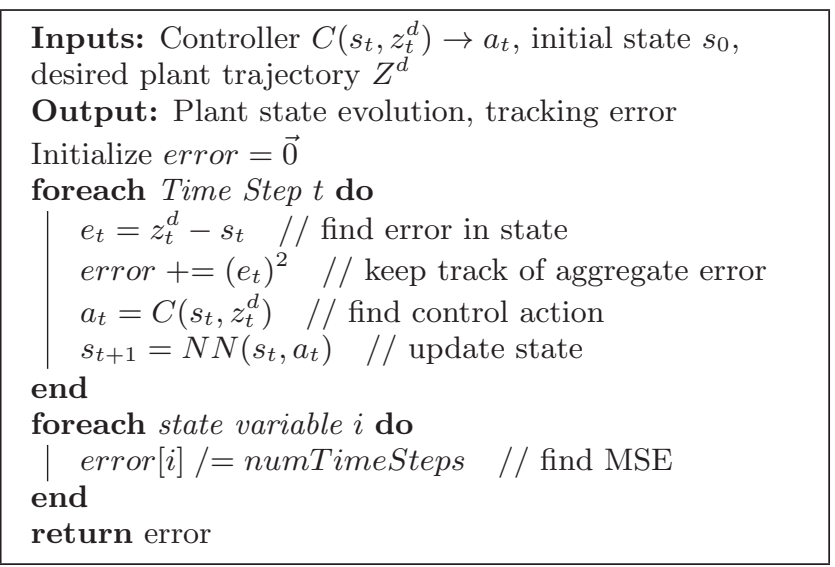

Algorithm 2: Time Domain Simulation

simulator takes an initial plant state $s_{0}$, a desired plant trajectory $Z^{d}$, and a controller $C\left(s_{t}, z_{t}^{d}\right) \rightarrow a_{t}$. The controller maps the current plant state $s_{t}$ and the desired plant state $z_{t}^{d}$ to a control action $a_{t}$. The simulator outputs the mean squared error of each state variable with respect to the desired plant trajectory. The time domain simulation is detailed in Algorithm 2.

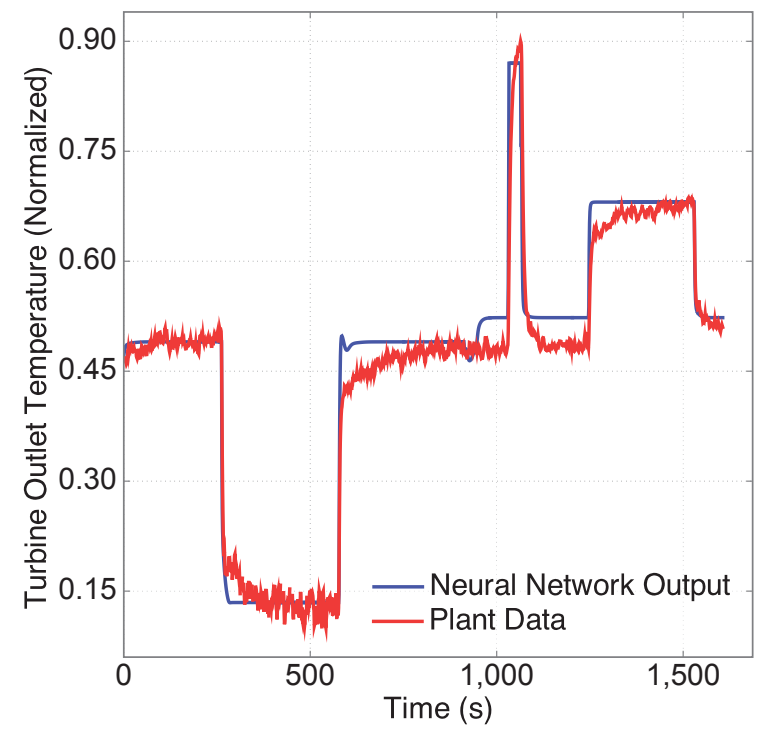

Figure 4: Comparison of true plant data with the simulation for turbine outlet temperature

To validate the Hyper simulator, the simulation is given the initial plant state from the original data and then allowed to respond to the control inputs from the original data. Figure 4 shows the response of the system to the original control inputs found in the data. Mean squared error across all plant state variables is $0.121 \%$. As can be seen in Figure 4, the maximum error of the network approximation corresponds to about $5 \%$ at any point in the simulation for turbine outlet temperature. This error is acceptable for developing controllers, because it is of a similar magnitude compared to noise inherent in turbine temperature sensors. This simulator along with desired Hyper system state trajec-

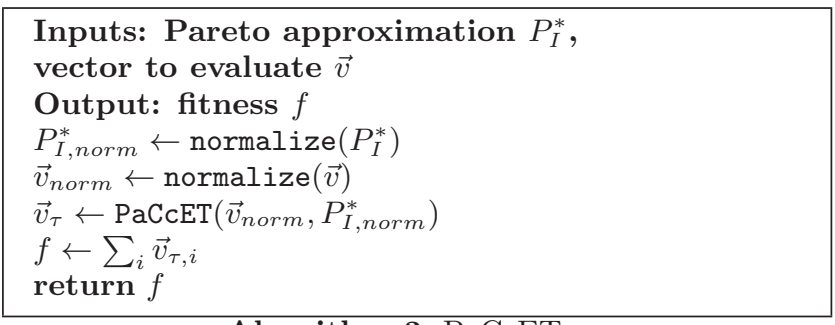

Algorithm 3: PaCcET

tories allows us to quickly evaluate the fitness of controllers in a neuroevolutionary algorithm.

\section{MULTI-OBJECTIVE NEUROEVOLUTION- ARY CONTROL ALGORITHM}

The following sections detail the Pareto Concavity Elimination Transformation ( $\mathrm{PaCcET}$ ) algorithm used for multiobjective fitness assignment, as well as the multi-objective neuroevolutionary control algorithm used in this work.

\subsection{PaCcET}

The full details of PaCcET are provided in [22]. In this section we provide an overview of the algorithm. For a more detailed treatment, we refer the reader to the other works on PaCcET $[22,23]$.

Determining the fitness of a two-objective solution with PaCcET requires $P_{I}^{*}$, the current best-estimate of the Pareto front, and the solution $v$ to be examined. For each solution, the PaCcET module finds the boundaries of the Pareto front, and normalizes $P_{I, \text { norm }}^{*} \leftarrow \operatorname{normalize}\left(P_{I}^{*}\right)$, for which each point in $P_{I}^{*}$ will have each of their elements in the range $[0: 1]$. It then uses this same normalization for the vector in question, $v$ : $v_{\text {norm }} \leftarrow \operatorname{normalize}(v)$

Once these normalizations have been completed, PaCcET then transforms $P_{I}^{*}$ to be on line between $(0,1)$ and $(1,0)$. This line is equally valuable to a linear combination of transformed objectives. Using this same transform for $v$ : $v_{\tau} \leftarrow$ $\operatorname{PaCcET}\left(v_{\text {norm }}\right)$, fitness $\leftarrow \sum_{i} v_{\tau, i}$ will be less than 1 if the solution is non-dominated, and greater than 1 if it is dominated.

After evaluating the fitness, $P_{I}^{*}$ must be maintained, and as the estimate is updated, it will continually improve to be a better approximation of the true Pareto front.

\subsection{Neuroevolutionary Control Algorithm}

The multi-objective neural network controllers for Hyper are evolved according to the neuroevolutionary algorithm presented in Algorithm 4. The neuroevolutionary algorithm corresponds to Box 5 in Figure 1, while the scalarization of performance across multiple objectives to assign a fitness (Box 4 in Figure 1) is achieved via the PaCcET algorithm. Throughout the evolutionary algorithm, the NonDominated Set (NDS, $P_{I}^{*}$ ) is updated to keep all non-dominated solutions found during learning.

The neural network controllers are two-layer, fully connected, feedforward neural networks. The networks have 38 inputs (corresponding to 19 state variables and 19 desired state variables), 10 hidden units, and 2 outputs (corresponding to the control actions for the bleed air valve and cold air valve). $N$ Neural networks are initialized by drawing weights from the normal distribution $N\left(\sigma_{\text {init }}, \mu_{\text {init }}\right)$, where the stan- 
dard deviation for weight initialization $\sigma_{\text {init }}$ is set to 0.5 and the mean for weight initialization $\mu_{\text {init }}$ is set to 0.0 .

For each generation, we implement a mutation operator, find the vector fitness (multi-objective fitness values) for each network, scalarize the multi-objective fitness vectors and assign fitness to each network, and downselect the population. These operators are now described in detail. For mutation, the population size is doubled from $N$ to $2 N$ by creating a mutated copy of each of the $N$ networks in the population. To mutate a network, 10 weights in each weight layer are selected at random, and values drawn from the normal distribution $N\left(\sigma_{\text {mutate }}, \mu_{\text {mutate }}\right.$ are added to each selected weight. For the normal distribution corresponding to mutation, the standard deviation $\sigma_{\text {mutate }}$ is set to 1.0 , and the mean $\mu_{\text {mutate }}$ is set to 0.0 . Learning parameters (number of hidden units, $\left.N, \sigma_{\text {init }}, \mu_{\text {init }}, \sigma_{\text {mutate }}, \mu_{\text {mutate }}\right)$ were chosen using a parameter sweep, but results were fairly consistent as long as the number of hidden units was above 5 .

To assign fitness, each of the $2 N$ neural network controllers are tested in the Hyper simulation, and their performance at tracking a desired plant trajectory are determined. The simulator gives a vector of mean squared error values, one for each state variable in the plant. Next, $w$ different state variables are chosen as the parameters of interest; these are the different objectives being optimized by the neuroevolutionary algorithm and correspond to critical state variables in the Hyper facility. The mean squared error $M S E_{i}$ associated with each of these parameters of interest $i$ are used to compute fitness values according to:

$$
f i t_{i}=\frac{1.0}{1.0+M S E_{i}}
$$

Once the vector fitness (one fitness value for each objective) is found, this vector is used as an input to the PaCcET algorithm, which converts the vector fitness into a scalar value. This scalar is used to assign fitness to a network.

After each of the $2 N$ networks are assigned scalar fitness values, $N$ networks are selected to survive to the next generation. For selection, a binary tournament operator is used. Two networks are taken from the population at random, and the network with a higher fitness value is placed into the population for the next generation. At the end of each generation, the NDS is updated by adding any population members to the NDS that are not dominated by any solutions in the NDS. Once the maximum number of generations is reached, the evolutionary algorithm returns the NDS, or the collection of neural network controllers along the approximated Pareto front.

\section{RESULTS}

For the experimental analysis of the neuroevolutionary multi-objective control algorithm for Hyper, we chose two objectives. The first objective corresponds to tracking a desired turbine speed profile, which has various oscillations corresponding to load demands. The second objective corresponds to keeping the fuel cell temperature as steady as possible, in order to minimize the transient operation of the fuel cell. In other words, we aim to develop a controller that simultaneously tracks load demands and keeps the fuel cell as close to steady-state as possible. For the experiments, we ran 50 statistical trials of the neuroevolutionary algorithm. For each trial, the population size $N$ was set to 25 , and each

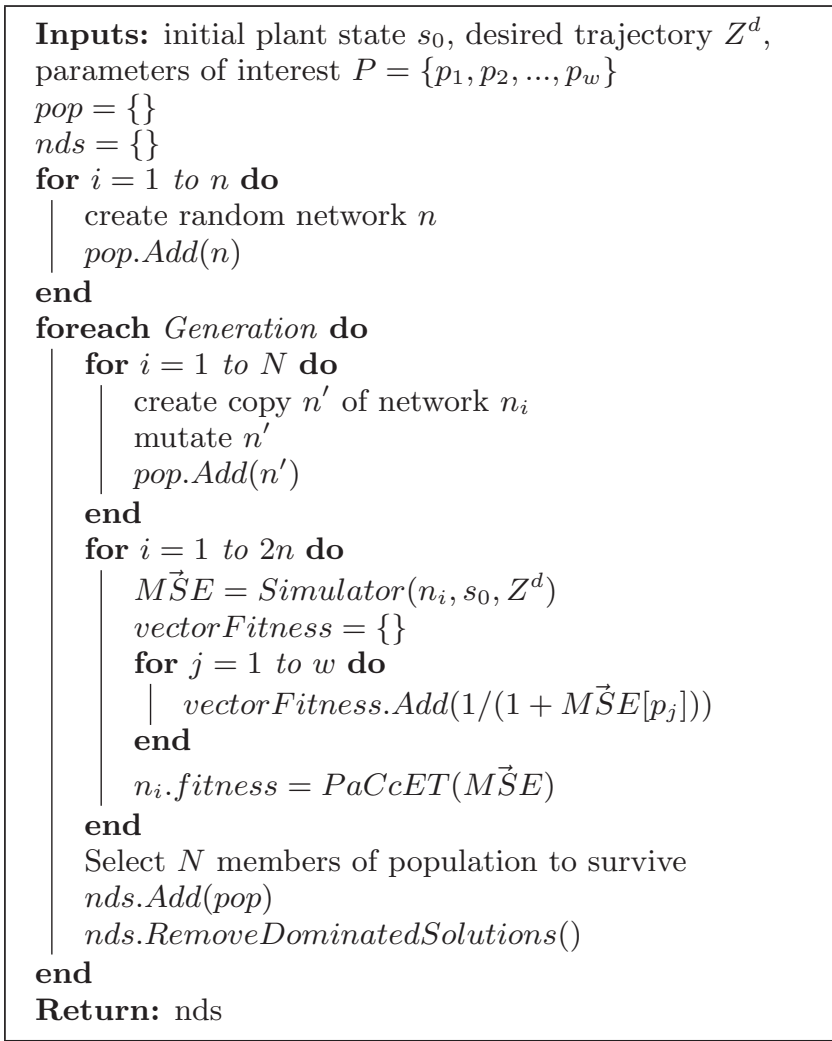

Algorithm 4: Neuroevolutionary algorithm to develop Hyper controllers.

evolutionary algorithm was run for 7500 generations.

The empirical attainment functions for the non-dominated sets are shown in Figure 5. This figure suggests that the Pareto front may have a strongly concave shape. The tradeoff solutions near the middle of the front were found in nearly every trial, but the extremes were found in less than half.

We now consider the two policies corresponding to the endpoints of the median NDS in Figure 5. The policy on the upper left of the NDS corresponds to maximizing the steady state operation of the fuel cell without considering turbine speed, while the policy on the lower right of the NDS corresponds to optimizing turbine speed tracking without considering fuel cell temperature. The performance of these policies on turbine tracking are shown in Figure 6.

As seen in Figure 6, the policy which maximized turbine tracking accuracy tracks the desired turbine speed well, while the policy which maximized the time the fuel cell spent in steady state does not track the desired turbine speed well. Instead of accurate turbine tracking, this policy keeps the turbine at a higher than needed speed. Although this is wasteful, this policy does ensure that load demands would always be met. The performance of these two policies on the fuel cell temperature are shown in Figure 7.

As seen in Figure 7, the policy which optimized turbine tracking accuracy does very poorly at keeping the fuel cell operation near steady state. This is because the fuel cell and turbine are extremely coupled, making it difficult to track a dynamic turbine speed while keeping the fuel cell temperature constant. Conversely, the policy that aimed to keep the fuel cell operating at steady state does very well, 


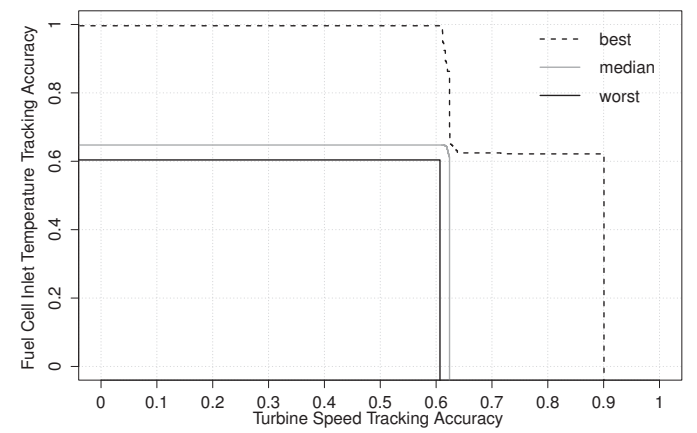

Figure 5: Empirical Attainment Function for multiobjective controllers. "Best:" collection of nondominated points developed across all statistical runs. "Median:" area which was attained in half of the statistical runs. "Worst:" area attained by every statistical run.

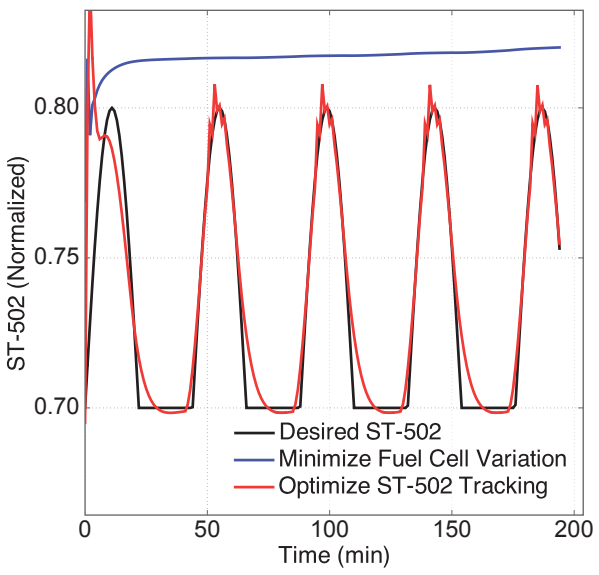

Figure 6: Pareto front endpoint control policies for turbine speed.

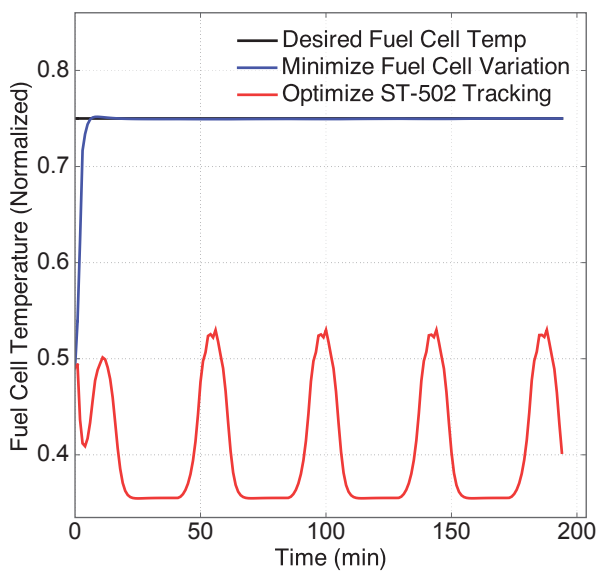

Figure 7: Endpoint control policies for fuel cell temperature. keeping the fuel cell temperature essentially constant after the initial heating period.

We now analyze policies along the interior of the nondominated set, which represent tradeoffs between the objectives. Figures 8 and 9 show three tradeoff policies and their performances in tracking desired turbine and fuel cell trajectories, respectively. The green curves show high oscillation in both the turbine speed and fuel cell temperatures, demonstrating that policies which appear viable mathematically (based on the fitness function) may not result in desirable behaviors on a physical system. The orange curves show a policy which does not track the desired turbine speed well (although does normally meet or exceed output requirements), and keeps the fuel cell closer to steady state. Finally, the magenta curve simply exceeds the desired turbine speed at all times (ensuring output requirements are always met), and keeps the fuel cell at essentially steady state operation. These tradeoffs demonstrate that after finding a non-dominated set of control policies, a system designer can choose which option is best suited for application in the physical system.

\section{DISCUSSION}

Increased energy demands as well as the desire for more sustainable energy production have led to the development of more efficient energy sources. One such source is a fuel cell hybrid energy system, where a synergistic relationship between the fuel cell and turbine power cycle result in increased efficiency, decreased emissions, and faster transient plant responses. A key difficulty in such plants is the high nonlinearities and coupling, resulting the the development of models to be extremely difficult.

In this work, we implement a model-free approach to developing multi-objective controllers for the Hyper plant using neuroevolution. In order for the evolutionary algorithm to be computationally tractable, we developed a statistical simulator of the Hyper facility based on data from physical runs. This simulator was combined with PaCcET to form a multi-objective fitness assignment operator.

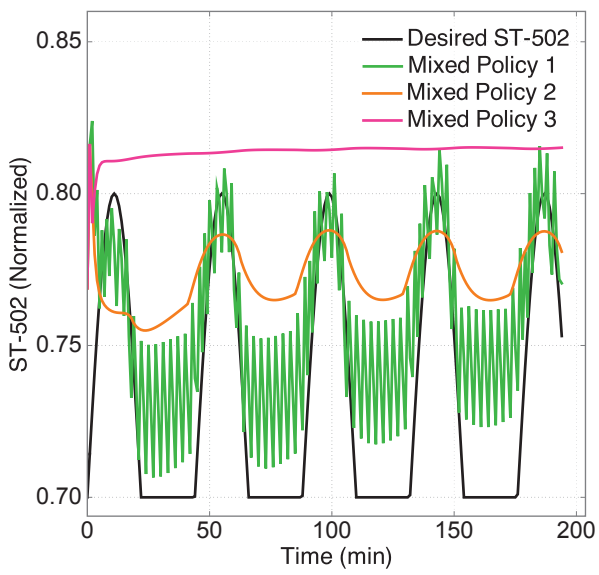

Figure 8: Tradeoff control policies for turbine speed

Our results demonstrate that a set of non-dominated controllers can be found that either provide excellent performance on single objectives, or provide tradeoff choices in which the objectives are balanced. 
Future work involves refining and validating the simulator to allow for better system modeling, followed by hardware tests of the derived control policies at the Hyper facility.

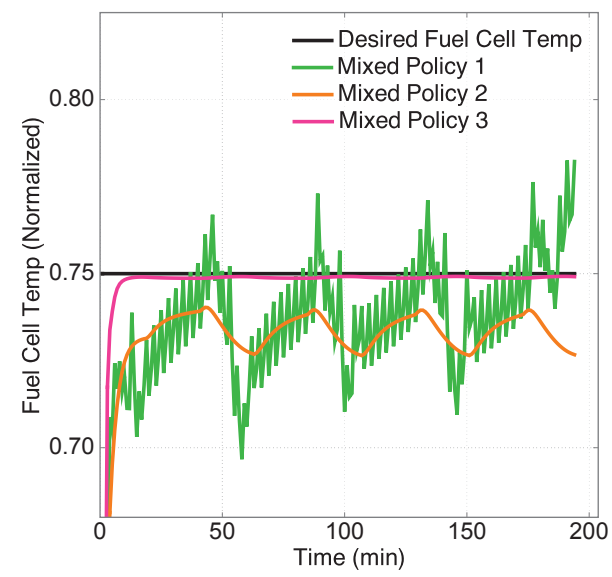

Figure 9: Tradeoff control policies for fuel cell temperature

\section{ACKNOWLEDGEMENTS}

This research was supported in part by the US Department of Energy - Office of Fossil Energy under Contract No. DE-AC02-07CH11358 through the Ames Laboratory. It was also supported in part by the US Department of Energy National Energy Technology Laboratory under Contract No. DE-FE0012302.

\section{REFERENCES}

[1] K. Anderson and Y. Hsu. Genetic crossover strategy using an approximation concept. In IEEE Congress on Evolutionary Computation, 1999.

[2] J. Branke, C. Schmidt, and H. Schmeck. Efficient fitness estimation in noisy environments. In Proceedings of the Genetic and Evolutionary Computation Conference, 2001.

[3] D. Bunche, N. Schraudolph, and P. Koumoutsakos. Accelerating evolutionary algorithms using fitness function models. In Proceedings of the Genetic and Evolutionary Computation Conference, 2003.

[4] M. Colby, E. Nasroullahi, and K. Tumer. Optimizing ballast design of wave energy converters using evolutionary algorithms. In Proceedings of the Genetic and Evolutionary Computation Conference, 2011.

[5] F. Gomez and R. Miikkulainen. Active guidance for a finless rocket using neuroevolution. In Proceedings of the Genetic and Evolutionary Computation Conference, 2003.

[6] Y. Jin. A comprehensive survey of fitness approximation in evolutionary computation. Soft Computing, 9(1), 2005.

[7] J. Martikainen and S. Ovaska. Fitness function approximation by neural networks in the optimization of mgp-fir filters. In the 2006 IEEE Mountain Workshop on Adaptive and Learning Systems, 2006.

[8] P. Pezzini, D. Tucker, and A. Traverso. Avoiding compressor surge during emergency shutdown hyrbid turbine systems. Journal of Engineering for Gas Turbines and Power, 2015.

[9] S. Qin and T. Badgwell. A survey of industrial model predictive control technology. Control Engineering Practice, 11(7), 2003.

[10] M. Salichon and K. Tumer. A neuro-evolutionary approach to micro aerial vehicle control. In Proceedings of the Genetic and Evolutionary Computation Conference, 2010.

[11] J. Shepard and K. Tumer. Robust neuro-control for a micro quadrotor. In Proceedings of the Genetic and Evolutionary Computation Conference, 2010.

[12] T. Smitch, C. Haynes, W. Wepfer, D. Tucker, and E. Liese. Hardware-based simulation of a fuel cell turbine hybrid response to imposed fuel cell load transients. ASME 2006 International Mechanical Engineering Congress and Exposition, 2006.

[13] J. Spall and J. Cristion. Model-free control of nonlinear stochastic systems with discrete time measurements. IEEE Transactions on Automatic Control, 43(9), 1998.

[14] A. Traverso, D. Tucker, and C. Haymes. Preliminary experimental results of igfc operation using hardware simulation. ASME J. Eng. Gas Turbines Power, 134(7), 2011.

[15] A. Tsai, L. Banta, L. Lawson, and D. Tucker. Determination of an empirical transfer function of a solid oxide fuel cell gas turbine hybrid system via frequency response analysis. Journal of Fuel Cell Science and Technology, 6(3), 2009.

[16] A. Tsai, D. Tucker, and T. Emami. Adaptive control of a nonlinear fuel cell gas turbine balance of plant simulation facility. Journal of Fuel Cell Science and Technology, 11(6), 2014.

[17] D. Tucker, L. Lawson, and R. Gemmen. Preliminary result of a cold flow test in a fuel cell gas turbine hybrid simulation facility. ASME Turbo Expo, 2003.

[18] D. Tucker, L. Lawson, and R. Gemmen. Characterization of air flow management and control in a fuel cell turbine hybrid power system using hardware simulation. In ASME 2005 Power Conference, 2005.

[19] D. Tucker, M. Shelton, and A. Manivannan. The role of solid oxide fuel cells in advanced hybrid power systems of the future. The Electrochemical Society Interface, 18(3), 2009.

[20] D. A. V. Veldhuizen and G. B. Lamont. Multiobjective evolutionary algorithms: Analyzing the state-of-the-art. Evolutionary Computation, 8(2):125-147, 2000.

[21] W. Winkler, P. Nehter, M. Williams, D. Tucker, and R. Gemmen. General fuel cell hybrid synergies and hybrid system testing status. Journal of Power Sources, 159(1), 2006.

[22] L. Yliniemi and K. Tumer. PaCcET: An objective space transformation to iteratively convexify the pareto front. In 10th International Conference on Simulated Evolution And Learning (SEAL), 2014.

[23] L. Yliniemi and K. Tumer. Complete coverage in the multi-objective PaCcET framework. In S. Silva, editor, Genetic and Evolutionary Computation Conference, 2015. 